\title{
Fixed point theorems for the sum of three classes of mixed monotone operators and applications
}

\author{
Xinqiu Zhang ${ }^{1}$, Lishan $\mathrm{Liu}^{1,2^{*}}$ and Yonghong $\mathrm{Wu}^{2,3}$
}

\section{"Correspondence:} mathlls@163.com

'School of Mathematical Sciences, Qufu Normal University, Qufu, Shandong 273165, People's Republic of China

${ }^{2}$ Department of Mathematics and Statistics, Curtin University, Perth, WA 6845, Australia

Full list of author information is available at the end of the article

\begin{abstract}
In this paper we develop various new fixed point theorems for a class of operator equations with three general mixed monotone operators, namely

$A(x, x)+B(x, x)+C(x, x)=x$ on ordered Banach spaces, where $A, B, C$ are the mixed monotone operators. $A$ is such that for any $t \in(0,1)$, there exists $\varphi(t) \in(t, 1]$ such that for all $x, y \in P, A\left(t x, t^{-1} y\right) \geq \varphi(t) A(x, y) ; B$ is hypo-homogeneous, i.e. $B$ satisfies that for any $t \in(0,1), x, y \in P, B\left(t x, t^{-1} y\right) \geq t B(x, y) ; C$ is concave-convex, i.e. $C$ satisfies that for fixed $y, C(\cdot, y): P \rightarrow P$ is concave; for fixed $x, C(x, \cdot): P \rightarrow P$ is convex. Also we study the solution of the nonlinear eigenvalue equation $A(x, x)+B(x, x)+C(x, x)=\lambda x$ and discuss its dependency to the parameter. Our work extends many existing results in the field of study. As an application, we utilize the results obtained in this paper for the operator equation to study the existence and uniqueness of positive solutions for a class of nonlinear fractional differential equations with integral boundary conditions.
\end{abstract}

MSC: 47H07;47H14; 26A33; 34A08

Keywords: mixed monotone operator; $\varphi(t)$-concave-convex operator; concave-convex operator; hypo-homogeneous operator; fixed point theorem; fractional differential equation

\section{Introduction}

Mixed monotone operators are an important class of operators which are used intensively in engineering, nuclear physics, biology, chemistry, technology, etc. They were first introduced by Guo and Lakshmikantham in [1]. Thereafter, many authors have investigated this kind of operators in Banach spaces and obtained a lot of interesting and important results (see [2-10]).

In [11], Chen discussed the conditions which guarantee the existence of an asymptotically attractive fixed point for $T=A+H: P \rightarrow P$, where $P$ is a cone of a Banach space $E$. $A, H: P \rightarrow P$ are two monotone operators in $E$ and there exist $\eta>0$ and $\alpha \in(0,1)$ such that

$$
H(t x) \geq t H x, \quad \forall t \in(\eta, 1), x \in P
$$

and

$$
A(t x) \geq t^{\alpha} A x, \quad \forall t \in(\eta, 1), x \in P,
$$

(c) 2016 Zhang et al. This article is distributed under the terms of the Creative Commons Attribution 4.0 International License (http://creativecommons.org/licenses/by/4.0/), which permits unrestricted use, distribution, and reproduction in any medium, provided you give appropriate credit to the original author(s) and the source, provide a link to the Creative Commons license, and indicate if changes were made. 
where $\alpha \in(\eta, 1)$. If there is an $x_{0} \in P$ such that $A x_{0} \in C_{x_{0}}$ and $H x_{0} \in C_{x_{0}}$, then there exists $\lambda:(\eta, 1) \times C_{x_{0}} \rightarrow(\alpha, 1)$ such that $T(t x) \geq t^{\lambda(t, x)} T x, t \in(\eta, 1), x \in P$. Furthermore, if $\lambda_{m}(t)=$ $\sup _{x \in C_{x_{0}}} \lambda(t, x)<1$, then there exists $x_{*} \in C_{x_{0}}$ such that $\lim _{n \rightarrow \infty} T^{n} x_{0}=x_{*}$ and $T x_{*}=x_{*}$.

In [12], Zhang used the partial order theory to study mixed monotone operators which have different types of concave-convex properties (for example: $A(x, y)$ is concave in $x$, and $(-\alpha)$-convex in $y$ ). The author also assumes that there exist $u_{0}, v_{0} \in \stackrel{\circ}{P}, \varepsilon>0, \varepsilon \geq \alpha$ such that $0 \ll u_{0} \leq v_{0}, u_{0} \leq A\left(u_{0}, v_{0}\right), A\left(v_{0}, u_{0}\right) \leq v_{0}$; and $\left.A\left(\theta, v_{0}\right) \geq \varepsilon A\left(v_{0}, u_{0}\right)\right)$, and he establishes the existence and uniqueness of a fixed point without assuming the operator to be compact or continuous.

In [13], Zhai and Hao considered the existence and uniqueness of positive solutions to the operator equation $A(x, x)+B x=x$ on ordered Banach spaces, where $A$ is a mixed monotone operator, $B$ is an increasing sub-homogeneous operator or $\alpha$-concave operator, and assume that:

(1) there is $h_{0} \in P_{h}$ such that $A\left(h_{0}, h_{0}\right) \in P_{h}, B h_{0} \in P_{h}$;

(2) there exists a constant $\delta_{0}>0$, such that $A(x, y) \geq \delta_{0} B(x, y), \forall x, y \in P$.

In [14], Zhai and Anderson studied an operator equation $A x+B x+C x=x$ on ordered Banach spaces, where $A$ is an increasing $\alpha$-concave operator, $B$ is an increasing subhomogeneous operator and $C$ is a homogeneous operator and satisfy:

(1) there is $h>\theta$ such that $A h \in P_{h}, B h \in P_{h}, C h \in P_{h}$;

(2) there exist constants $\delta_{1}, \delta_{2}>0$, such that $A x \geq \delta_{1} B x+\delta_{2} C x, \forall x \in P$.

The existence and uniqueness of positive solutions of the operator equation is obtained by using the properties of cones and a fixed point theorem for increasing general $\beta$ concave operators.

Motivated by the above work, this paper studies the existence and uniqueness of positive solutions to the following operator equation on ordered Banach spaces:

$$
A(x, x)+B(x, x)+C(x, x)=x
$$

where $A, B, C: P \times P \rightarrow P$ are mixed monotone operators and satisfy the following conditions, respectively:

(1) for any $t \in(0,1)$, there exists $\varphi(t) \in(t, 1]$ such that

$$
A\left(t x, t^{-1} y\right) \geq \varphi(t) A(x, y), \quad \forall x, y \in P
$$

(2) for any $t \in(0,1)$,

$$
B\left(t x, t^{-1} y\right) \geq t B(x, y), \quad \forall x, y \in P
$$

(3) for any fixed $y, C(\cdot, y): P \rightarrow P$ is concave; for any fixed $x, C(x, \cdot): P \rightarrow P$ is convex.

Also we study the solution of the nonlinear eigenvalue equation $A(x, x)+B(x, x)+$ $C(x, x)=\lambda x$ and discuss its properties. As an application, we utilize the results obtained for the operator equations to study the existence and uniqueness of positive solutions for a class of nonlinear fractional differential equations with integral boundary conditions.

To our knowledge, so far no fixed point results have been achieved for the operator of equation (1.1) with the operators $A, B, C$ satisfying the above listed conditions (1), (2), and 
(3). Our work presented in this paper has various new features. First,by using the properties of cones, we obtain some existence and uniqueness results of positive solutions for the operator of equation (1.1) without the need of assuming the operators to be continuous or compact, which extends the corresponding results in [11-23]. Most importantly our work is about the sum of three classes of mixed monotone operators. We also discusses the solution of the nonlinear eigenvalue equation $A(x, x)+B(x, x)+C(x, x)=\lambda x$ and investigate its dependency to the parameter. To demonstrate the applicability of our abstract results, we give, in the last section of the paper, some applications to nonlinear fractional differential equations with integral boundary conditions, and also, we give some specific examples.

\section{Preliminaries and lemmas}

For convenience in the discussion of the following sections, we briefly present here some definitions, notations and known results. For more details, we refer the reader to $[1,16,19$, 24-26] and the references therein.

Suppose that $(E,\|\cdot\|)$ is a Banach space and $\theta$ is the zero element of $E$. Recall that a nonempty closed convex set $P \subset E$ is a cone if it satisfies (1) $x \in P, \lambda \geq 0 \Rightarrow \lambda x \in P$; (2) $x \in P$, $-x \in P \Rightarrow x=\theta$. The Banach space $E$ can be partially ordered by a cone $P \subset E$, i.e., $x \leq y$ if and only if $y-x \in P$. If $x \leq y$ and $x \neq y$, then we denote $x<y$ or $y>x$.

A cone $P$ is said to be solid if its interior $\stackrel{\circ}{P}$ is non-empty. If $x-y \in \stackrel{\circ}{P}$, then we denote $x \gg y$. Moreover, $P$ is called normal if there exists a constant $N>0$ such that for all $x, y \in E$, $\theta \leq x \leq y$ implies $\|x\| \leq N\|y\|$, where the smallest $N$ is called the normality constant of $P$. If $x_{1}, x_{2} \in E$, the set $\left[x_{1}, x_{2}\right]=\left\{x \in E \mid x_{1} \leq x \leq x_{2}\right\}$ is called the order interval between $x_{1}$ and $x_{2}$. We say that an operator $A: E \rightarrow E$ is increasing (decreasing) if $x \leq y$ implies $A x \leq A y(A y \leq A x)$. An operator $A: P \rightarrow P$ is said to be $\alpha$-concave if there exists $\alpha \in(0,1)$ such that for all $t \in(0,1), x \in P, A(t x) \geq t^{\alpha} A x$.

For $x, y \in E$, the notation $x \sim y$ means that there exist $\lambda>0$ and $\mu>0$ such that $\lambda x \leq y \leq$ $\mu x$. Clearly, $\sim$ is an equivalence relation. Given $h>0$, we denote by $P_{h}$ the set $P_{h}=\{x \in$ $P \mid x \sim h\}$. It is easy to see that $P_{h} \subset P$. If $P$ is a solid cone, take any $h \in \stackrel{\circ}{P}$, then $P_{h}=\stackrel{\circ}{P}$. For $x, y \in P_{h}$, we can define

$$
M(x / y)=\inf \{\lambda \in R: x \leq \lambda y\} .
$$

Definition 2.1 [1, 16] $A: P \times P \rightarrow P$ is said to be a mixed monotone operator if $A(x, y)$ is increasing in $x$, and decreasing in $y$, i.e., $u_{i}, v_{i}(i=1,2) \in P, u_{1} \leq u_{2}, v_{1} \geq v_{2}$ imply $A\left(u_{1}, v_{1}\right) \leq$ $A\left(u_{2}, v_{2}\right)$. An element $x \in P$ is called a fixed point of $A$ if $A(x, x)=x$.

Definition 2.2 [22] $A: D(A) \subset E \rightarrow E$ is said to be convex if for any $x, y \in D(A)$ with $x \leq y$ and every $t \in(0,1)$, we have $A(t x+(1-t) y) \leq t A x+(1-t) A y$. $A$ is said to be concave if $-A$ is convex.

\section{Main results}

In this section we consider the existence and uniqueness of positive solutions for the operator of equation (1.1). Throughout the paper, we assume that $E$ is a real Banach space with a partial order introduced by a normal cone $P$ of $E$. Take $h \in E, h>\theta, P_{h}$ is given as in the introduction. 
Theorem 3.1 Let $P$ be a normal cone in E. Assume that $A, B, C: P \times P \rightarrow P$ are three mixed monotone operators and satisfy the following conditions:

(1) for any $t \in(0,1)$, there exists $\varphi(t) \in(t, 1]$ such that

$$
A\left(t x, t^{-1} y\right) \geq \varphi(t) A(x, y), \quad \forall x, y \in P
$$

(2) for any $t \in(0,1), x, y \in P$,

$$
B\left(t x, t^{-1} y\right) \geq t B(x, y)
$$

(3) for any fixed $y \in P, C(\cdot, y): P \rightarrow P$ is concave; for any fixed $x \in P, C(x, \cdot): P \rightarrow P$ is convex;

(4) there is $h \in P, h>\theta$ such that $A(h, h) \in P_{h}, B(h, h) \in P_{h}$, and $C(h, h) \in P_{h}$;

(5) there exists $\frac{1}{2} \leq c \leq 1$, such that $C(\theta, l h) \geq c C(l h, \theta)$, for any $l \geq 1$;

(6) there exists a constant $\delta_{0}>0$, such that $B(x, y)+C(x, y) \leq \delta_{0} A(x, y), \forall x, y \in P_{h}$.

Then the operator of equation (1.1) has a unique positive solution $x^{*}$ in $P$, which satisfies $\mu h \leq x^{*} \leq \lambda h$, where $\lambda>0, \mu>0$ are two real numbers. And for any initial values $x_{0}, y_{0} \in$ $P_{h}$, by constructing successively the sequences as follows:

$$
\begin{aligned}
& x_{n}=A\left(x_{n-1}, y_{n-1}\right)+B\left(x_{n-1}, y_{n-1}\right)+C\left(x_{n-1}, y_{n-1}\right), \\
& y_{n}=A\left(y_{n-1}, x_{n-1}\right)+B\left(y_{n-1}, x_{n-1}\right)+C\left(y_{n-1}, x_{n-1}\right), \quad n=1,2, \ldots,
\end{aligned}
$$

we have $x_{n} \rightarrow x^{*}$ and $y_{n} \rightarrow x^{*}$ in $E$, as $n \rightarrow \infty$.

Proof From (3.1) and (3.2), for any $t \in(0,1)$, we have

$$
A\left(t^{-1} x, t y\right) \leq \frac{1}{\varphi(t)} A(x, y), \quad \forall x, y \in P
$$

and

$$
B\left(t^{-1} x, t y\right) \leq t^{-1} B(x, y), \quad \forall x, y \in P
$$

Since $A(h, h) \in P_{h}, B(h, h) \in P_{h}, C(h, h) \in P_{h}$, there exist constants $a_{i}>0, b_{i}>0(i=1,2,3)$ such that

$$
a_{1} h \leq A(h, h) \leq b_{1} h, \quad a_{2} h \leq B(h, h) \leq b_{2} h, \quad a_{3} h \leq C(h, h) \leq b_{3} h .
$$

First of all, we show $A: P_{h} \times P_{h} \rightarrow P_{h}$. For any $x, y \in P_{h}$, we can choose two sufficiently small numbers $\alpha_{1}, \alpha_{2} \in(0,1)$ such that

$$
\alpha_{1} h \leq x \leq \frac{1}{\alpha_{1}} h, \quad \alpha_{2} h \leq y \leq \frac{1}{\alpha_{2}} h .
$$

Let $\alpha=\min \left\{\alpha_{1}, \alpha_{2}\right\}$, then $\alpha \in(0,1)$, by (3.1), (3.3), and (3.4), we have

$$
A(x, y) \leq A\left(\frac{1}{\alpha} h, \alpha h\right) \leq \frac{1}{\varphi(\alpha)} A(h, h) \leq \frac{1}{\varphi(\alpha)} b_{1} h,
$$




$$
A(x, y) \geq A\left(\alpha h, \frac{1}{\alpha} h\right) \geq \varphi(\alpha) A(h, h) \geq \varphi(\alpha) a_{1} h
$$

Evidently, $\frac{1}{\varphi(\alpha)} b_{1}, \varphi(\alpha) a_{1}>0$. Thus $A(x, y) \in P_{h}$; that is, $A: P_{h} \times P_{h} \rightarrow P_{h}$.

Second, we show $B: P_{h} \times P_{h} \rightarrow P_{h}$. For any $x, y \in P_{h}$, we can choose two sufficiently small numbers $\beta_{1}, \beta_{2} \in(0,1)$ such that

$$
\beta_{1} h \leq x \leq \frac{1}{\beta_{1}} h, \quad \beta_{2} h \leq y \leq \frac{1}{\beta_{2}} h .
$$

Let $\beta=\min \left\{\beta_{1}, \beta_{2}\right\}$, then $\beta \in(0,1)$, by (3.2), (3.3), and (3.4), we have

$$
\begin{aligned}
& B(x, y) \leq B\left(\frac{1}{\beta} h, \beta h\right) \leq \frac{1}{\beta} B(h, h) \leq \frac{1}{\beta} b_{2} h, \\
& B(x, y) \geq B\left(\beta h, \frac{1}{\beta} h\right) \geq \beta B(h, h) \geq \beta a_{2} h .
\end{aligned}
$$

Evidently, $\frac{1}{\beta} b_{2}, \beta a_{2}>0$. Thus $B(x, y) \in P_{h}$; that is, $B: P_{h} \times P_{h} \rightarrow P_{h}$.

Thirdly, we show $C: P_{h} \times P_{h} \rightarrow P_{h}$. For any $t \in(0,1), x, y \in P_{h}$, we have

$$
C(x, y)=C\left(x, t t^{-1} y+(1-t) \theta\right) \leq t C\left(x, t^{-1} y\right)+(1-t) C(x, \theta),
$$

thus

$$
t C\left(x, t^{-1} y\right) \geq C(x, y)-(1-t) C(x, \theta) .
$$

Also, we can find a sufficiently large $l$ such that $x, y, t^{-1} y \leq l h$ and satisfies (5), so from (5), (3.5), and the concavity and convexity as well as the monotone property of operator $C$, we have

$$
\begin{aligned}
C\left(t x, t^{-1} y\right) & \geq t C\left(x, t^{-1} y\right)+(1-t) C\left(\theta, t^{-1} y\right) \\
& \geq C(x, y)-(1-t) C(x, \theta)+(1-t) C(\theta, l h) \\
& \geq C(x, y)+(1-t)[C(\theta, l h)-C(l h, \theta)] \\
& \geq C(x, y)+(1-t)\left[C(\theta, l h)-\frac{1}{c} C(\theta, l h)\right] \\
& =C(x, y)+(1-t)\left(1-\frac{1}{c}\right) C(\theta, l h) \\
& \geq\left[1+(1-t)\left(1-\frac{1}{c}\right)\right] C(x, y) \\
& =\left[\left(2-\frac{1}{c}\right)+\left(\frac{1}{c}-1\right) t\right] C(x, y) \\
& \geq t C(x, y) .
\end{aligned}
$$

That is,

$$
C\left(t x, t^{-1} y\right) \geq t C(x, y), \quad \forall t \in(0,1), \forall x, y \in P_{h} .
$$


And then it is obvious that

$$
C\left(t^{-1} x, t y\right) \leq \frac{1}{t} C(x, y), \quad \forall t \in(0,1), \forall x, y \in P_{h} .
$$

Since $x, y \in P_{h}$, we can choose two sufficiently small numbers $\gamma_{1}, \gamma_{2} \in(0,1)$ such that

$$
\gamma_{1} h \leq x \leq \frac{1}{\gamma_{1}} h, \quad \gamma_{2} h \leq y \leq \frac{1}{\gamma_{2}} h .
$$

Let $\gamma=\min \left\{\gamma_{1}, \gamma_{2}\right\}$, then $\gamma \in(0,1)$, by (3.4), (3.6), and (3.7), we have

$$
\begin{aligned}
& C(x, y) \leq C\left(\frac{1}{\gamma} h, \gamma h\right) \leq \frac{1}{\gamma} C(h, h) \leq \frac{1}{\gamma} b_{3} h, \\
& C(x, y) \geq C\left(\gamma h, \frac{1}{\gamma} h\right) \geq \gamma C(h, h) \geq \gamma a_{3} h .
\end{aligned}
$$

Evidently, $\frac{1}{\gamma} b_{3}, \gamma a_{3}>0$. Thus $C(x, y) \in P_{h}$; that is, $C: P_{h} \times P_{h} \rightarrow P_{h}$.

Now we define an operator $T=A+B+C: P_{h} \times P_{h} \rightarrow P_{h}$ by

$$
T(x, y)=A(x, y)+B(x, y)+C(x, y), \quad x, y \in P_{h} .
$$

Then $T: P_{h} \times P_{h} \rightarrow P_{h}$ is a mixed monotone operator and $T(h, h) \in P_{h}$.

In the following, we show that for any $x, y \in P_{h}, t \in(0,1)$, there exists $\psi(t, x, y) \in(t, 1]$ such that $T\left(t x, t^{-1} y\right) \geq \psi(t, x, y) T(x, y)$. From (3.1), (3.2), and (3.6), for any $t \in(0,1)$ and $x, y \in P_{h}$, we have

$$
\begin{aligned}
T\left(t x, t^{-1} y\right) & =A\left(t x, t^{-1} y\right)+B\left(t x, t^{-1} y\right)+C\left(t x, t^{-1} y\right) \\
& \geq \varphi(t) A(x, y)+t B(x, y)+t C(x, y) \\
& \geq \varphi(t) A(x, y)+t[B(x, y)+C(x, y)]
\end{aligned}
$$

For any $x, y \in P_{h}$, since $A, B, C: P_{h} \times P_{h} \rightarrow P_{h}$, we have $A(x, y) \in P_{h}, B(x, y) \in P_{h}, C(x, y) \in P_{h}$, and thus we get $B(x, y)+C(x, y) \sim A(x, y)$. Denote

$$
F(x, y)=M\left(\frac{B(x, y)+C(x, y)}{A(x, y)}\right) .
$$

Then from (6) we have $F(x, y) \leq \delta_{0}$. For any $t \in(0,1), x, y \in P_{h}$, consider

$$
g(s)=\frac{\varphi(t)+F(x, y) t}{(F(x, y)+1) s}, \quad s \in[t, \varphi(t)] .
$$

It is clear that $g$ is continuous and strictly decreasing with respect to $s$. Since

$$
g\left(\frac{\delta_{0} t+\varphi(t)}{\delta_{0}+1}\right)=\frac{\varphi(t)+F(x, y) t}{(F(x, y)+1) \frac{\delta_{0} t+\varphi(t)}{\delta_{0}+1}}>1
$$

and

$$
g(\varphi(t))=\frac{\varphi(t)+F(x, y) t}{(F(x, y)+1) \varphi(t)}<1,
$$


there exists a unique $\psi(t, x, y) \in\left(\frac{\delta_{0} t+\varphi(t)}{\delta_{0}+1}, \varphi(t)\right)$ such that

$$
g(\psi(t, x, y))=\frac{\varphi(t)+F(x, y) t}{(F(x, y)+1) \psi(t, x, y)}=1
$$

Solving for $F(x, y)$ in the above inequality leads to

$$
F(x, y)=\frac{\varphi(t)-\psi(t, x, y)}{\psi(t, x, y)-t} .
$$

From (2.1) we get $M(x / y)=\inf \{\lambda \in R: x \leq \lambda y\}$. So from the definitions of $F(x, y)$ and $M(x / y)$, we can get

$$
B(x, y)+C(x, y) \leq \frac{\varphi(t)-\psi(t, x, y)}{\psi(t, x, y)-t} A(x, y) .
$$

This inequality can be rewritten as

$$
\varphi(t) A(x, y)+t[B(x, y)+C(x, y)] \geq \psi(t, x, y)[A(x, y)+B(x, y)+C(x, y)]
$$

Hence

$$
\begin{aligned}
T\left(t x, t^{-1} y\right) & =A\left(t x, t^{-1} y\right)+B\left(t x, t^{-1} y\right)+C\left(t x, t^{-1} y\right) \\
& \geq \varphi(t) A(x, y)+t B(x, y)+t C(x, y) \\
& =\varphi(t) A(x, y)+t[B(x, y)+C(x, y)] \\
& \geq \psi(t, x, y)[A(x, y)+B(x, y)+C(x, y)] \\
& =\psi(t, x, y) T(x, y) .
\end{aligned}
$$

That is, for any $x, y \in P_{h}$ and $t \in(0,1)$, there exists $\psi(t, x, y) \in\left(\frac{\delta_{0} t+\varphi(t)}{\delta_{0}+1}, \varphi(t)\right) \subseteq(t, 1]$ such that

$$
T\left(t x, t^{-1} y\right) \geq \psi(t, x, y) T(x, y)
$$

Since $T(h, h) \in P_{h}$, we can choose a sufficiently small number $s_{0} \in(0,1)$ such that

$$
s_{0} h \leq T(h, h) \leq \frac{1}{s_{0}} h .
$$

Noting that $s_{0}<\psi\left(s_{0}, x, y\right) \leq 1$, we can get $1<\frac{\psi\left(s_{0}, x, y\right)}{s_{0}} \leq \frac{1}{s_{0}}$. By the Archimedes principle, we can take a positive integer $k$ such that

$$
\left(\frac{\psi\left(s_{0}, x, y\right)}{s_{0}}\right)^{k} \geq \frac{1}{s_{0}}
$$

that is,

$$
\frac{\psi\left(s_{0}, x, y\right)}{s_{0}} \geq\left(\frac{1}{s_{0}}\right)^{\frac{1}{k}}
$$


Put $u_{0}=s_{0}^{k} h, v_{0}=s_{0}^{-k} h$. Evidently, $u_{0}, v_{0} \in P_{h}$ and $u_{0}=s_{0}^{2 k} v_{0}<v_{0}$. Take any $r \in\left(0, s_{0}^{2 k}\right]$, then $r \in(0,1)$ and $u_{0} \geq r v_{0}$. By the mixed monotone properties of $T$, we have $T\left(u_{0}, v_{0}\right) \leq$ $T\left(v_{0}, u_{0}\right)$. Further, by combining condition (3.8) with (3.9) and (3.10), we have

$$
\begin{aligned}
T\left(u_{0}, v_{0}\right) & =T\left(s_{0}^{k} h, \frac{1}{s_{0}^{k}} h\right) \\
& =T\left(s_{0} s_{0}^{k-1} h, \frac{1}{s_{0}} \frac{1}{s_{0}^{k-1}} h\right) \\
& \geq \psi\left(s_{0}, s_{0}^{k-1} h, \frac{1}{s_{0}^{k-1}} h\right) T\left(s_{0}^{k-1} h, \frac{1}{s_{0}^{k-1}} h\right) \\
& =\psi\left(s_{0}, s_{0}^{k-1} h, \frac{1}{s_{0}^{k-1}} h\right) T\left(s_{0} s_{0}^{k-2} h, \frac{1}{s_{0}} \frac{1}{s_{0}^{k-2}} h\right) \\
& =\psi\left(s_{0}, s_{0}^{k-1} h, \frac{1}{s_{0}^{k-1}} h\right) \psi\left(s_{0}, s_{0}^{k-2} h, \frac{1}{s_{0}^{k-2}} h\right) T\left(s_{0}^{k-2} h, \frac{1}{s_{0}^{k-2}} h\right) \geq \cdots \\
& \geq\left(\left(\frac{1}{s_{0}}\right)^{\frac{1}{k}} s_{0}\right)^{k} T(h, h) \\
& \geq \frac{1}{s_{0}} s_{0}^{k} s_{0} h \\
& \geq s_{0}^{k} h \\
& =u_{0} .
\end{aligned}
$$

From (3.8), we can get, for all $t \in(0,1), x, y \in P, T\left(t^{-1} x, t y\right) \leq \frac{1}{\psi(t, x, y)} T(x, y)$. So

$$
\begin{aligned}
T\left(v_{0}, u_{0}\right) & =T\left(\frac{1}{s_{0}^{k}} h, s_{0}^{k} h\right) \\
& =T\left(\frac{1}{s_{0}} \frac{1}{s_{0}^{k-1}} h, s_{0} s_{0}^{k-1} h\right) \\
& \leq \frac{1}{\psi\left(s_{0}, \frac{1}{s_{0}^{k-1}} h, s_{0}^{k-1} h\right)} T\left(\frac{1}{s_{0}^{k-1}} h, s_{0}^{k-1} h\right) \\
& =\frac{1}{\psi\left(s_{0}, \frac{1}{s_{0}^{k-1}} h, s_{0}^{k-1} h\right)} T\left(\frac{1}{s_{0}} \frac{1}{s_{0}^{k-2}} h, s_{0} s_{0}^{k-2} h\right) \\
& =\frac{1}{\psi\left(s_{0}, \frac{1}{s_{0}^{k-1}} h, s_{0}^{k-1} h\right)} \frac{1}{\psi\left(s_{0}, \frac{1}{s_{0}^{k-2}} h, s_{0}^{k-2} h\right)} T\left(\frac{1}{s_{0}^{k-2}} h, s_{0}^{k-2} h\right) \leq \cdots \\
& \leq\left(\frac{1}{s_{0}} s_{0}^{\frac{1}{k}}\right)^{k} T(h, h) \\
& \leq \frac{1}{s_{0}^{k}} s_{0} \frac{1}{s_{0}} h \\
& \leq \frac{1}{s_{0}^{k}} h \\
& =v_{0} .
\end{aligned}
$$


Thus we have

$$
u_{0} \leq T\left(u_{0}, v_{0}\right) \leq T\left(v_{0}, u_{0}\right) \leq v_{0}
$$

For $u_{0}, v_{0}$, construct successively the sequences as follows:

$$
u_{n}=T\left(u_{n-1}, v_{n-1}\right), \quad v_{n}=T\left(v_{n-1}, u_{n-1}\right), \quad n=1,2, \ldots
$$

Evidently, $u_{1} \leq v_{1}$. By the mixed monotone properties of $T$, we obtain $u_{n} \leq v_{n}(n=1,2, \ldots)$ and

$$
u_{0} \leq u_{1} \leq \cdots \leq u_{n} \leq \cdots \leq v_{n} \leq \cdots \leq v_{1} \leq v_{0}
$$

Noting that $u_{0} \geq r v_{0}$, we can get $u_{n} \geq u_{0} \geq r v_{0} \geq r v_{n}(n=1,2, \ldots)$. Let

$$
t_{n}=\sup \left\{t>0 \mid u_{n} \geq t v_{n}, n=1,2, \ldots\right\}
$$

Then we have

$$
u_{n} \geq t_{n} v_{n}, \quad n=1,2, \ldots
$$

and then by (3.11) we have

$$
u_{n+1} \geq u_{n} \geq t_{n} v_{n} \geq t_{n} v_{n+1}, \quad n=1,2, \ldots
$$

Therefore, $t_{n+1} \geq t_{n}$, i.e., $\left\{t_{n}\right\}$ is increasing with $\left\{t_{n}\right\} \subset(0,1]$. Suppose $t_{n} \rightarrow t^{*}$ as $n \rightarrow \infty$, then $t^{*}=1$. Otherwise, $0<t^{*}<1$. Since $t_{n} \leq t^{*}$ and $\psi(t, x, y)>t$, by the mixed monotone properties of $T$ and (3.8) as well as (3.12), we have

$$
\begin{aligned}
u_{n+1} & =T\left(u_{n}, v_{n}\right) \\
& \geq T\left(t_{n} v_{n}, \frac{1}{t_{n}} u_{n}\right) \\
& =T\left(\frac{t_{n}}{t^{*}} t^{*} v_{n}, \frac{t^{*}}{t_{n}} \frac{1}{t^{*}} u_{n}\right) \\
& \geq \frac{t_{n}}{t^{*}} T\left(t^{*} v_{n}, \frac{1}{t^{*}} u_{n}\right) \\
& \geq \frac{t_{n}}{t^{*}} \psi\left(t^{*}, v_{n}, u_{n}\right) T\left(v_{n}, u_{n}\right) \\
& =\frac{t_{n}}{t^{*}} \psi\left(t^{*}, v_{n}, u_{n}\right) v_{n+1} .
\end{aligned}
$$

By the definition of $t_{n}$, we have $t_{n+1} \geq \frac{t_{n}}{t^{*}} \psi\left(t^{*}, v_{n}, u_{n}\right)$, that is, $\psi\left(t^{*}, v_{n}, u_{n}\right) \leq \frac{t^{*}}{t_{n}} t_{n+1}$. So we get

$$
t^{*}<\frac{\delta_{0} t^{*}+\varphi\left(t^{*}\right)}{\delta_{0}+1}<\psi\left(t^{*}, v_{n}, u_{n}\right) \leq \frac{t^{*}}{t_{n}} t_{n+1}, \quad n=1,2, \ldots
$$


Since $\lim _{n \rightarrow \infty} \frac{t^{*}}{t_{n}} t_{n+1}=t^{*}$, we get $t^{*}<\frac{\delta_{0} t^{*}+\varphi\left(t^{*}\right)}{\delta_{0}+1} \leq t^{*}$, which is a contradiction. Thus, $\lim _{n \rightarrow \infty} t_{n}=1$. For any natural number $p$ we have

$$
\begin{aligned}
& \theta \leq u_{n+p}-u_{n} \leq v_{n}-u_{n} \leq v_{n}-t_{n} v_{n}=\left(1-t_{n}\right) v_{n} \leq\left(1-t_{n}\right) v_{0}, \\
& \theta \leq v_{n}-v_{n+p} \leq v_{n}-u_{n} \leq\left(1-t_{n}\right) v_{0}, \quad n=1,2, \ldots
\end{aligned}
$$

Since the cone $P$ is normal, we have

$$
\left\|u_{n+p}-u_{n}\right\| \leq N\left(1-t_{n}\right)\left\|v_{0}\right\|, \quad\left\|v_{n}-v_{n+p}\right\| \leq N\left(1-t_{n}\right)\left\|v_{0}\right\|, \quad n, p=1,2, \ldots
$$

where $N$ is the normality constant of $P$. So we can claim that $\left\{u_{n}\right\}$ and $\left\{v_{n}\right\}$ are Cauchy sequences. Because $E$ is complete, there exist $u^{*}, v^{*}$ such that $u_{n} \rightarrow u^{*}, v_{n} \rightarrow v^{*}$ as $n \rightarrow \infty$. By (3.11), we know that $u_{n} \leq u^{*} \leq v^{*} \leq v_{n}$ with $u^{*}, v^{*} \in P_{h}$, and

$$
\theta \leq v^{*}-u^{*} \leq v_{n}-u_{n} \leq\left(1-t_{n}\right) v_{0}
$$

Further, by the normality of cone $P$, we have

$$
\left\|v^{*}-u^{*}\right\| \leq N\left(1-t_{n}\right)\left\|v_{0}\right\| \rightarrow 0, \quad n \rightarrow \infty
$$

and thus $u^{*}=v^{*}$. Let $x^{*}:=u^{*}=v^{*}$ and then by the mixed monotone properties of $T$, we obtain

$$
u_{n+1}=T\left(u_{n}, v_{n}\right) \leq T\left(x^{*}, x^{*}\right) \leq T\left(v_{n}, u_{n}\right)=v_{n+1}, \quad n=1,2,3, \ldots
$$

Let $n \rightarrow \infty$, then we get $x^{*}=T\left(x^{*}, x^{*}\right)$. That is, $x^{*}$ is a fixed point of $T$ in $P_{h}$.

In the following, we prove that $x^{*}$ is the unique fixed point of $T$ in $P_{h}$. In fact, suppose $\bar{x}$ is another fixed point of $T$ in $P_{h}$ and $\bar{x} \neq x^{*}$. Since $x^{*}, \bar{x} \in P_{h}$, there exist positive numbers $\mu_{1}, \mu_{2}, \lambda_{1}, \lambda_{2}>0$ such that

$$
\mu_{1} h \leq x^{*} \leq \lambda_{1} h, \quad \mu_{2} h \leq \bar{x} \leq \lambda_{2} h
$$

Then we obtain

$$
\bar{x} \leq \lambda_{2} h=\frac{\lambda_{2}}{\mu_{1}} \mu_{1} h \leq \frac{\lambda_{2}}{\mu_{1}} x^{*}, \quad \bar{x} \geq \bar{\mu}_{2} h=\frac{\mu_{2}}{\lambda_{1}} \lambda_{1} h \geq \frac{\mu_{2}}{\lambda_{1}} x^{*} .
$$

Let

$$
e_{1}=\sup \left\{t>0 \mid t x^{*} \leq \bar{x} \leq t^{-1} x^{*}\right\}
$$

Evidently, $0<e_{1} \leq 1, e_{1} x^{*} \leq \bar{x} \leq \frac{1}{e_{1}} x^{*}$. Next we prove $e_{1}=1$. If $0<e_{1}<1$, then by the mixed monotone properties of $T$ and (3.8), we would get

$$
\bar{x}=T(\bar{x}, \bar{x}) \geq T\left(e_{1} x^{*}, \frac{1}{e_{1}} x^{*}\right) \geq \psi\left(e_{1}, x^{*}, x^{*}\right) T\left(x^{*}, x^{*}\right)=\psi\left(e_{1}, x^{*}, x^{*}\right) x^{*}
$$

Since $\psi\left(e_{1}, x^{*}, x^{*}\right)>e_{1}$, this contradicts the definition of $e_{1}$, so we get $e_{1}=1$. Thus $\bar{x}=x^{*}$. Therefore, $A$ has a unique fixed point $x^{*}$ in $P_{h}$. 
Now we construct successively the sequences

$$
x_{n}=T\left(x_{n-1}, y_{n-1}\right), \quad y_{n}=T\left(y_{n-1}, x_{n-1}\right), \quad n=1,2, \ldots,
$$

for any initial points $x_{0}, y_{0} \in P_{h}$. Since $x_{0}, y_{0} \in P_{h}$, we can choose small numbers $e_{2}, e_{3} \in$ $(0,1)$ such that

$$
e_{2} h \leq x_{0} \leq \frac{1}{e_{2}} h, \quad e_{3} h \leq y_{0} \leq \frac{1}{e_{3}} h .
$$

Let $e_{*}=\min \left\{e_{2}, e_{3}\right\}$. Then $e_{*} \in(0,1)$ and

$$
e_{*} h \leq x_{0}, y_{0} \leq \frac{1}{e_{*}} h
$$

Since $e_{*}<\psi\left(e_{*}, x, y\right) \leq 1$, we can get $1<\frac{\psi\left(e_{*}, x, y\right)}{e_{*}} \leq \frac{1}{e_{*}}$. By the Archimedes principle, we can choose a sufficiently large positive integer $m$ such that

$$
\frac{\psi\left(e_{*}, x, y\right)}{e_{*}} \geq\left(\frac{1}{e_{*}}\right)^{\frac{1}{m}}
$$

Put $\bar{u}_{0}=e_{*}^{m} h, \bar{v}_{0}=\frac{1}{e_{*}^{m}} h$. It is easy to see that $\bar{u}_{0}, \bar{v}_{0} \in P_{h}$, and $\bar{u}_{0}<x_{0}, y_{0}<\bar{v}_{0}$. Let

$$
\bar{u}_{n}=T\left(\bar{u}_{n-1}, \bar{v}_{n-1}\right), \quad \bar{v}_{n}=T\left(\bar{v}_{n-1}, \bar{u}_{n-1}\right), \quad n=1,2, \ldots
$$

Similarly, it follows that there exists $y^{*} \in P_{h}$ such that

$$
T\left(y^{*}, y^{*}\right)=y^{*}, \quad \lim _{n \rightarrow \infty} \bar{u}_{n}=\lim _{n \rightarrow \infty} \bar{v}_{n}=y^{*}
$$

By the uniqueness of the fixed points of the operator $T$ in $P_{h}$, we get $x^{*}=y^{*}$. And by induction, $\bar{u}_{n} \leq x_{n}, y_{n} \leq \bar{v}_{n}(n=1,2, \ldots)$. Since the cone $P$ is normal, we have $\lim _{n \rightarrow \infty} x_{n}=$ $\lim _{n \rightarrow \infty} y_{n}=x^{*}$.

Remark 3.1 Theorem 3.1 is a fixed point theorem for the sum of three classes of mixed monotone operators, which extends the results in [11-14].

Taking $B, C=\theta$ in Theorem 3.1, we get the following corollary.

Corollary 3.1 Let P be a normal cone in E. Assume that $T: P \times P \rightarrow P$ is a mixed monotone operator and satisfies the following conditions:

$\left(\mathrm{A}_{1}\right)$ There exists $h \in P$ with $h>\theta$ such that $T(h, h) \in P_{h}$.

$\left(\mathrm{A}_{2}\right)$ For any $t \in(0,1)$, there exists $\varphi(t) \in(t, 1]$ such that

$$
T\left(t x, t^{-1} y\right) \geq \varphi(t) T(x, y), \quad \forall x, y \in P
$$

Then the operator $T(x, x)=x$ has a unique solution $x^{*}$ in $P$, which satisfies $\mu h \leq x^{*} \leq \lambda h$, where $\lambda>0, \mu>0$ are two real numbers. Moreover, for any initial values $x_{0}, y_{0} \in P_{h}$, by 
constructing successively the sequences as follows:

$$
x_{n}=T\left(x_{n-1}, y_{n-1}\right), \quad y_{n}=T\left(y_{n-1}, x_{n-1}\right), \quad n=1,2, \ldots,
$$

we have $x_{n} \rightarrow x^{*}$ and $y_{n} \rightarrow x^{*}$ in $E$, as $n \rightarrow \infty$.

Proof Since $X$ is a uniformly convex Banach space and $A$ is bounded, we see that $A_{0}$ is non-empty and $\left\{(P T)^{n}(x)\right\}$ is bounded for any $x \in A_{0}$. By Theorem 3.1, $T$ has at least one best proximity point.

Remark 3.2 Under the conditions $\left(\mathrm{A}_{1}\right),\left(\mathrm{A}_{2}\right)$, this corollary not only guarantees the existence of upper-lower solutions for the operator $T$ and the existence of a unique fixed point, but also it constructs successively some sequences for approximating the fixed point.

Taking $A, B=\theta$ and $\frac{1}{2}<c \leq 1$ in Theorem 3.1, from the proof of Theorem 3.1 we get the following corollary.

Corollary 3.2 Let $P$ be a normal cone in E. Assume that $C: P \times P \rightarrow P$ is a mixed monotone operator and satisfies the following conditions:

(1) for any fixed $y \in P, C(\cdot, y): P \rightarrow P$ is concave; for any fixed $x \in P, C(x, \cdot): P \rightarrow P$ is convex;

(2) there is $h \in P, h>\theta$ such that $C(h, h) \in P_{h}$;

(3) there exists $\frac{1}{2}<c \leq 1$, such that $C(\theta, l h) \geq c C(l h, \theta), l \geq 1$.

Then the operator $C(x, x)=x$ has a unique solution $x^{*}$ in $P$, which satisfies $\mu h \leq x^{*} \leq \lambda h$, where $\lambda>0, \mu>0$ are two real numbers. Furthermore, for any initial values $x_{0}, y_{0} \in P_{h}$, by constructing successively the sequences as follows:

$$
x_{n}=C\left(x_{n-1}, y_{n-1}\right), \quad y_{n}=C\left(y_{n-1}, x_{n-1}\right), \quad n=1,2, \ldots,
$$

we have $x_{n} \rightarrow x^{*}$ and $y_{n} \rightarrow x^{*}$ in $E$, as $n \rightarrow \infty$.

Remark 3.3 In the above corollary we do not need to require the operator $C$ to satisfy $0<C(\theta, v) \leq v$, which extends the result in [12].

Taking $\varphi(t)=t^{\alpha}, \alpha \in(0,1)$, we get the following corollary, which generalizes and improves Theorem 2.1 in [21].

Corollary 3.3 Let $P$ be a normal cone in $E, \alpha \in(0,1)$. Assume that $A, B, C: P \times P \rightarrow P$ are three mixed monotone operators and satisfy the following conditions:

(1) for any $t \in(0,1), x, y \in P$, we have $A\left(t x, t^{-1} y\right) \geq t^{\alpha} A(x, y)$;

(2) for any $t \in(0,1), x, y \in P$, we have $B\left(t x, t^{-1} y\right) \geq t B(x, y)$;

(3) for any fixed $y \in P, C(\cdot, y): P \rightarrow P$ is concave; for any fixed $x \in P, C(x, \cdot): P \rightarrow P$ is convex;

(4) there is $h \in P, h>\theta$ such that $A(h, h) \in P_{h}, B(h, h) \in P_{h}$, and $C(h, h) \in P_{h}$;

(5) there exists $\frac{1}{2} \leq c \leq 1$, such that $C(\theta, l h) \geq c C(l h, \theta)$, for any $l \geq 1$;

(6) there exists a constant $\delta_{0}>0$, such that $B(x, y)+C(x, y) \leq \delta_{0} A(x, y), \forall x, y \in P_{h}$.

Then the operator equation (1.1) has a unique solution $x^{*}$ in $P$, which satisfies $\mu h \leq x^{*} \leq$ $\lambda h$, where $\lambda>0, \mu>0$ are two real numbers. Furthermore, for any initial values $x_{0}, y_{0} \in P_{h}$, 
by constructing successively the sequences as follows:

$$
\begin{aligned}
& x_{n}=A\left(x_{n-1}, y_{n-1}\right)+B\left(x_{n-1}, y_{n-1}\right)+C\left(x_{n-1}, y_{n-1}\right), \\
& y_{n}=A\left(y_{n-1}, x_{n-1}\right)+B\left(y_{n-1}, x_{n-1}\right)+C\left(y_{n-1}, x_{n-1}\right), \quad n=1,2, \ldots,
\end{aligned}
$$

we have $x_{n} \rightarrow x^{*}$ and $y_{n} \rightarrow x^{*}$ as $n \rightarrow \infty$.

Corollary 3.4 Let $P$ be a normal cone in E. Let $h>0$ and $A, B, C: P_{h} \times P_{h} \rightarrow P_{h}$ are three mixed monotone operators and satisfy the following conditions:

(1) for any $t \in(0,1)$, there exists $\varphi(t) \in(t, 1]$ such that

$$
A\left(t x, t^{-1} y\right) \geq \varphi(t) A(x, y), \quad \forall x, y \in P_{h}
$$

(2) for any $t \in(0,1), x, y \in P_{h}, B\left(t x, t^{-1} y\right) \geq t B(x, y)$;

(3) for any fixed $y \in P_{h}, C(\cdot, y): P_{h} \rightarrow P_{h}$ is concave; for any fixed $x \in P_{h}$, $C(x, \cdot): P_{h} \rightarrow P_{h}$ is convex;

(4) there exists $\frac{1}{2} \leq c \leq 1$, such that $C(\theta, l h) \geq c C(l h, \theta), l \geq 1$;

(5) there exists a constant $\delta_{0}>0$, such that $B(x, y)+C(x, y) \leq \delta_{0} A(x, y), \forall x, y \in P_{h}$.

Then the operator of equation (1.1) has a unique solution $x^{*}$ in $P$, which satisfies $\mu h \leq$ $x^{*} \leq \lambda h$, where $\lambda>0, \mu>0$ are two real numbers. And for any initial values $x_{0}, y_{0} \in P_{h}$, by constructing successively the sequences as follows:

$$
\begin{aligned}
& x_{n}=A\left(x_{n-1}, y_{n-1}\right)+B\left(x_{n-1}, y_{n-1}\right)+C\left(x_{n-1}, y_{n-1}\right), \\
& y_{n}=A\left(y_{n-1}, x_{n-1}\right)+B\left(y_{n-1}, x_{n-1}\right)+C\left(y_{n-1}, x_{n-1}\right), \quad n=1,2, \ldots,
\end{aligned}
$$

we have $x_{n} \rightarrow x^{*}$ and $y_{n} \rightarrow x^{*}$ as $n \rightarrow \infty$.

Remark 3.4 If $P$ is a solid cone, $h \in \stackrel{\circ}{P}$. If we suppose that the operators $A, B, C: P_{h} \times P_{h} \rightarrow$ $P_{h}$ or $A, B, C: \stackrel{\circ}{P} \times \stackrel{\circ}{P} \rightarrow \stackrel{\circ}{P}$, then $A(h, h) \in P_{h}, B(h, h) \in P_{h}$, and $C(h, h) \in P_{h}$ are automatically satisfied in Corollary 3.4 .

Theorem 3.2 Let $P$ be a normal cone in E. A, B, C:P $P \rightarrow P$ are three mixed monotone operators and satisfy the following conditions:

(1) for any $t \in(0,1)$, there exists $\varphi(t) \in(t, 1]$ such that

$$
A\left(t x, t^{-1} y\right) \geq \varphi(t) A(x, y), \quad \forall x, y \in P
$$

(2) for any $t \in(0,1), x, y \in P, B\left(t x, t^{-1} y\right) \geq t B(x, y)$;

(3) for any fixed $y \in P, A(\cdot, y): P \rightarrow P$ is concave; for any fixed $x \in P, A(x, \cdot): P \rightarrow P$ is convex;

(4) there is $h \in P, h>\theta$ such that $A(h, h) \in P_{h}, B(h, h) \in P_{h}$, and $C(h, h) \in P_{h}$;

(5) there exists $\frac{1}{2} \leq c \leq 1$, such that $C(\theta, l h) \geq c C(l h, \theta), l \geq 1$;

(6) there exists a constant $\delta_{0}>0$, such that $B(x, y)+C(x, y) \leq \delta_{0} A(x, y), \forall x, y \in P_{h}$.

Then for any given $\lambda>0$, the operator equation

$$
A(x, x)+B(x, x)+C(x, x)=\lambda x
$$


has a unique solution $x_{\lambda}$ in $P$, which satisfies $\mu h \leq x_{\lambda} \leq \lambda h$, where $\lambda>0, \mu>0$ are two real numbers. Furthermore, we have the following conclusions:

(R1) if $\varphi(t)>t^{\frac{1}{2}}\left(\delta_{0}+1\right)-\delta_{0} t$ for $t \in(0,1)$, then $x_{\lambda}$ is strictly decreasing in $\lambda$, that is, $0<\lambda_{1}<\lambda_{2}$ implies $x_{\lambda_{1}}>x_{\lambda_{2}}$;

(R2) if there exists $\beta \in(0,1)$ such that $\varphi(t) \geq t^{\beta}\left(\delta_{0}+1\right)-\delta_{0} t$ for $t \in(0,1)$, then $x_{\lambda}$ is continuous in $\lambda$, that is, $\lambda \rightarrow \lambda_{0}\left(\lambda_{0}>0\right)$ implies $\left\|x_{\lambda}-x_{\lambda_{0}}\right\| \rightarrow 0$;

(R3) if there exists $\beta \in\left(0, \frac{1}{2}\right)$ such that $\varphi(t) \geq t^{\beta}\left(\delta_{0}+1\right)-\delta_{0} t$ for $t \in(0,1)$, then $\lim _{\lambda \rightarrow \infty}\left\|x_{\lambda}\right\|=0, \lim _{\lambda \rightarrow 0^{+}}\left\|x_{\lambda}\right\|=\infty$.

Proof For fixed $\lambda>0$, by Theorem 3.1, $\frac{1}{\lambda} T: P_{h} \times P_{h} \rightarrow P_{h}$ is mixed monotone and satisfies

$$
\left(\frac{1}{\lambda} T\right)\left(t x, t^{-1} y\right)=\frac{1}{\lambda} T\left(t x, t^{-1} y\right) \geq \frac{1}{\lambda} \psi(t, x, y) T(x, y)=\psi(t, x, y)\left(\frac{1}{\lambda} T\right)(x, y),
$$

for any $x, y \in P_{h}, t \in(0,1)$. So it follows from Theorem 3.1 that $\frac{1}{\lambda} T$ has a unique fixed point $x_{\lambda}$ in $P_{h}$. That is, $T\left(x_{\lambda}, x_{\lambda}\right)=\lambda x_{\lambda}$. For convenience of proof, we let

$$
\alpha(t, x, y)=\frac{\ln \psi(t, x, y)}{\ln t}, \quad \forall t \in(0,1) .
$$

Then $\alpha(t, x, y) \in[0,1)$ and $\psi(t, x, y)=t^{\alpha(t, x, y)}$. Thus $T\left(t x, t^{-1} y\right) \geq t^{\alpha(t, x, y)} T(x, y)$, for any $x, y \in$ $P_{h}, t \in(0,1)$.

(1) Proof of (R1). Suppose $0<\lambda_{1}<\lambda_{2}$ and let

$$
t_{0}=\sup \left\{t>0 \mid x_{\lambda_{1}} \geq t x_{\lambda_{2}}, x_{\lambda_{2}} \geq t x_{\lambda_{1}}\right\}
$$

then we have $0<t_{0}<1$ and

$$
x_{\lambda_{1}} \geq t_{0} x_{\lambda_{2}}, \quad x_{\lambda_{2}} \geq t_{0} x_{\lambda_{1}} .
$$

By the mixed monotone properties of $T$,

$$
\begin{aligned}
& \lambda_{1} x_{\lambda_{1}}=T\left(x_{\lambda_{1}}, x_{\lambda_{1}}\right) \geq T\left(t_{0} x_{\lambda_{2}}, t_{0}^{-1} x_{\lambda_{2}}\right) \geq t_{0}^{\alpha\left(t_{0}, x_{\lambda_{2}}, x_{\lambda_{2}}\right)} T\left(x_{\lambda_{2}}, x_{\lambda_{2}}\right)=t_{0}^{\alpha\left(t_{0}, x_{\lambda_{2}}, x_{\lambda_{2}}\right)} \lambda_{2} x_{\lambda_{2}}, \\
& \lambda_{2} x_{\lambda_{2}}=T\left(x_{\lambda_{2}}, x_{\lambda_{2}}\right) \geq T\left(t_{0} x_{\lambda_{1}}, t_{0}^{-1} x_{\lambda_{1}}\right) \geq t_{0}^{\alpha\left(t_{0}, x_{\lambda_{1}}, x_{\lambda_{1}}\right)} T\left(x_{\lambda_{1}}, x_{\lambda_{1}}\right)=t_{0}^{\alpha\left(t_{0}, x_{\lambda_{1}}, x_{\lambda_{1}}\right)} \lambda_{1} x_{\lambda_{1}} .
\end{aligned}
$$

Further

$$
x_{\lambda_{1}} \geq \lambda_{1}^{-1} \lambda_{2} t_{0}^{\alpha\left(t_{0}, x_{\lambda_{2}}, x_{\lambda_{2}}\right)} x_{\lambda_{2}}, \quad x_{\lambda_{2}} \geq \lambda_{2}^{-1} \lambda_{1} t_{0}^{\alpha\left(t_{0}, x_{\lambda_{1}}, x_{\lambda_{1}}\right)} x_{\lambda_{1}} .
$$

Noting that $\lambda_{1}^{-1} \lambda_{2} t_{0}^{\alpha\left(t_{0}, x_{\lambda_{2}}, x_{\lambda_{2}}\right)}>t_{0}$, from the definition of $t_{0}$ and (3.14), we get

$$
\lambda_{2}^{-1} \lambda_{1} t_{0}^{\alpha\left(t_{0}, x_{\lambda_{1}}, x_{\lambda_{1}}\right)} \leq t_{0}
$$

which in turn yields

$$
t_{0} \geq\left(\frac{\lambda_{1}}{\lambda_{2}}\right)^{\frac{1}{1-\alpha\left(t_{0}, x_{\lambda_{1}}, x_{\lambda_{1}}\right)}} .
$$


Hence

$$
x_{\lambda_{1}} \geq \lambda_{1}^{-1} \lambda_{2}\left(\frac{\lambda_{1}}{\lambda_{2}}\right)^{\frac{\alpha\left(t_{0}, x_{2}, x_{\lambda_{2}}\right)}{1-\alpha\left(t_{0}, \lambda_{1}, \lambda_{\lambda_{1}}\right)}} x_{\lambda_{2}}=\left(\frac{\lambda_{2}}{\lambda_{1}}\right)^{\frac{1-2 \alpha\left(t_{0}, x_{\lambda_{1}}, x_{\lambda_{1}}\right)}{1-\alpha\left(t_{0}, x_{\lambda_{2}}, \lambda_{\lambda_{2}}\right)}} x_{\lambda_{2}} .
$$

Noting that $\varphi\left(t_{0}\right)>t_{0}^{\frac{1}{2}}\left(\delta_{0}+1\right)-\delta_{0} t_{0}$, we have $\psi\left(t_{0}, x, y\right)>\frac{\varphi\left(t_{0}\right)+\delta_{0} t_{0}}{\delta_{0}+1}>t_{0}^{\frac{1}{2}}$, and thus we have $\alpha\left(t_{0}, x, y\right)<\frac{1}{2}$ and consequently,

$$
\left(\frac{\lambda_{2}}{\lambda_{1}}\right)^{\frac{1-2 \alpha\left(t_{0}, x_{\lambda_{1}}, x_{\lambda_{1}}\right)}{1-\alpha\left(t_{0}, x_{\lambda_{2}}, x_{2}\right)}}>1
$$

Thus, $x_{\lambda_{1}}>x_{\lambda_{2}}$.

(2) Proof of (R2). Since $\varphi(t) \geq t^{\beta}\left(\delta_{0}+1\right)-\delta_{0} t$ for $t \in(0,1)$, we have $\psi(t, x, y)>\frac{\varphi\left(t_{0}\right)+\delta_{0} t_{0}}{\delta_{0}+1} \geq$ $t^{\beta}$ for $t \in(0,1)$, and thus we get $\alpha(t, x, y) \leq \beta$ for $t \in(0,1)$. By (3.13) and (3.15), we have

$$
\begin{aligned}
\left(\frac{\lambda_{1}}{\lambda_{2}}\right)^{\frac{1}{1-\beta}} x_{\lambda_{2}} & \leq\left(\frac{\lambda_{1}}{\lambda_{2}}\right)^{\frac{1}{1-\alpha\left(t_{0}, x_{\lambda_{1}}, x_{\lambda_{1}}\right)}} x_{\lambda_{2}} \leq x_{\lambda_{1}} \\
& \leq \frac{1}{t_{0}} x_{\lambda_{2}} \leq\left(\frac{\lambda_{2}}{\lambda_{1}}\right)^{\frac{1}{1-\alpha\left(t_{0}, x_{\lambda_{1}}, x_{\lambda_{1}}\right)}} x_{\lambda_{2}} \leq\left(\frac{\lambda_{2}}{\lambda_{1}}\right)^{\frac{1}{1-\beta}} x_{\lambda_{2}}, \\
\left(\frac{\lambda_{1}}{\lambda_{2}}\right)^{\frac{1}{1-\beta}} x_{\lambda_{1}} & \leq\left(\frac{\lambda_{1}}{\lambda_{2}}\right)^{\frac{1}{1-\alpha\left(t_{0}, x_{\lambda_{1}}, x_{\lambda_{1}}\right)}} x_{\lambda_{1}} \leq x_{\lambda_{2}} \leq \frac{1}{t_{0}} x_{\lambda_{1}} \\
& \leq\left(\frac{\lambda_{2}}{\lambda_{1}}\right)^{\frac{1}{1-\alpha\left(t_{0}, x_{\lambda_{1}}, x_{\lambda_{1}}\right)}} x_{\lambda_{1}} \leq\left(\frac{\lambda_{2}}{\lambda_{1}}\right)^{\frac{1}{1-\beta}} x_{\lambda_{1}} .
\end{aligned}
$$

Further

$$
\theta \leq x_{\lambda_{1}}-\left(\frac{\lambda_{1}}{\lambda_{2}}\right)^{\frac{1}{1-\beta}} x_{\lambda_{2}} \leq\left[\left(\frac{\lambda_{2}}{\lambda_{1}}\right)^{\frac{1}{1-\beta}}-\left(\frac{\lambda_{1}}{\lambda_{2}}\right)^{\frac{1}{1-\beta}}\right] x_{\lambda_{2}}
$$

Consequently, from the normality of cone $P$ and (3.17), we get

$$
\begin{aligned}
\left\|x_{\lambda_{1}}-x_{\lambda_{2}}\right\| & \leq\left\|x_{\lambda_{1}}-\left(\frac{\lambda_{1}}{\lambda_{2}}\right)^{\frac{1}{1-\beta}} x_{\lambda_{2}}\right\|+\left\|\left(\frac{\lambda_{1}}{\lambda_{2}}\right)^{\frac{1}{1-\beta}} x_{\lambda_{2}}-x_{\lambda_{2}}\right\| \\
& \leq N\left[\left(\frac{\lambda_{2}}{\lambda_{1}}\right)^{\frac{1}{1-\beta}}-\left(\frac{\lambda_{1}}{\lambda_{2}}\right)^{\frac{1}{1-\beta}}\right]\left\|x_{\lambda_{2}}\right\|+\left|\left(\frac{\lambda_{1}}{\lambda_{2}}\right)^{\frac{1}{1-\beta}}-1\right|\left\|x_{\lambda_{2}}\right\|,
\end{aligned}
$$

where $N$ is the normality constant. Let $\lambda_{1} \rightarrow \lambda_{2}^{-}$, we have $\left\|x_{\lambda_{1}}-x_{\lambda_{2}}\right\| \rightarrow 0$. Similarly, let $\lambda_{2} \rightarrow \lambda_{1}^{+}$, from (3.18) we can also prove $\left\|x_{\lambda_{2}}-x_{\lambda_{1}}\right\| \rightarrow 0$. So the conclusion (R2) holds.

(3) Proof of (R3). Since $\varphi(t) \geq t^{\beta}\left(\delta_{0}+1\right)-\delta_{0} t$ for $t \in(0,1)$, we have $\psi(t, x, y)>\frac{\varphi\left(t_{0}\right)+\delta_{0} t_{0}}{\delta_{0}+1} \geq$ $t^{\beta}$ for $t \in(0,1)$, and thus we have $\alpha(t, x, y) \leq \beta<\frac{1}{2}$ for $t \in(0,1)$. Let $\lambda_{1}=1, \lambda_{2}=\lambda$ in (3.16), then we have

$$
x_{1} \geq \lambda^{\frac{1-2 \alpha\left(t_{0}, x_{1}, x_{1}\right)}{1-\alpha\left(t_{0}, x_{\lambda}, x_{\lambda}\right)}} x_{\lambda} \geq \lambda^{\frac{1-2 \beta}{1-\beta}} x_{\lambda}, \quad \forall \lambda>1 .
$$


Thus we can easily obtain

$$
\left\|x_{\lambda}\right\| \leq \frac{N}{\lambda^{\frac{1-2 \beta}{1-\beta}}}\left\|x_{1}\right\|, \quad \forall \lambda>1,
$$

where $N$ is the normality constant. Let $\lambda \rightarrow \infty$, then $\left\|x_{\lambda}\right\| \rightarrow 0$. Similarly, let $\lambda_{1}=\lambda, \lambda_{2}=1$ in (3.16), then

$$
x_{\lambda} \geq \lambda^{-\frac{1-2 \alpha\left(t_{0}, x_{\lambda}, x_{\lambda}\right)}{1-\alpha\left(t_{0}, x_{1}, x_{1}\right)}} x_{1} \geq \lambda^{-\frac{1-2 \beta}{1-\beta}} x_{1}, \quad \forall 0<\lambda<1 .
$$

Thus

$$
\left\|x_{\lambda}\right\| \geq N^{-1} \lambda^{-\frac{1-2 \beta}{1-\beta}}\left\|x_{1}\right\|, \quad \forall 0<\lambda<1,
$$

where $N$ is the normality constant. Let $\lambda \rightarrow 0^{+}$, then we have $\left\|x_{\lambda}\right\| \rightarrow \infty$.

\section{Applications}

Many problems in various areas, such as differential equations, integral equations, boundary value problems and nonlinear matrix equations, can be converted to the operator equation (1.1). We refer the reader to [27-34] and the references therein. In this section, we apply the results in Section 3 to study a class of nonlinear fractional differential equations with integral boundary conditions. We focus on the existence and uniqueness of positive solutions for the following nonlinear fractional differential equations with integral boundary conditions:

$$
\left\{\begin{array}{l}
D_{0+}^{\alpha} u(t)=f(t, u(t), u(t))+g(t, u(t), u(t))+k(t, u(t), u(t)), \quad 0<t<1, \\
u(0)=u^{\prime}(0)=u^{\prime \prime}(0)=0, \\
u(1)=\int_{0}^{\eta} u(s) d s
\end{array}\right.
$$

where $3<\alpha \leq 4,0<\eta<1$, and $D_{0_{+}}^{\alpha}$ is the Riemann-Liouville fractional derivative of order $\alpha>0$, defined by

$$
D_{0+}^{\alpha} u(t)=\frac{1}{\Gamma(n-\alpha)}\left(\frac{d}{d t}\right)^{n} \int_{0}^{t}(t-\tau)^{n-\alpha-1} u(\tau) d \tau .
$$

In the following, for the sake of convenience, set $E=C[0,1]$, the Banach space of continuous functions on $[0,1]$ with the norm $\|y\|=\max \{|y(t)|: t \in[0,1]\} . P=\{y \in C[0,1] \mid y(t) \geq$ $0, t \in[0,1]\}$. It is clear that $P$ is a normal cone of which the normality constant is 1 . The Green function of problem (4.1) is as follows:

$$
G(t, s)=\frac{1}{p(0) \Gamma(\alpha)}\left\{\begin{array}{l}
t^{\alpha-1}(1-s)^{\alpha-1}-\frac{(\eta-s)^{\alpha}}{\alpha} t^{\alpha-1}-\left(1-\frac{\eta^{\alpha}}{\alpha}\right)(t-s)^{\alpha-1}, \\
\quad 0 \leq s \leq t \leq 1, s \leq \eta ; \\
t^{\alpha-1}(1-s)^{\alpha-1}-\left(1-\frac{\eta^{\alpha}}{\alpha}\right)(t-s)^{\alpha-1}, \quad 0 \leq \eta \leq s \leq t \leq 1 ; \\
t^{\alpha-1}(1-s)^{\alpha-1}-\frac{(\eta-s)^{\alpha}}{\alpha} t^{\alpha-1}, \quad 0 \leq t \leq s \leq \eta \leq 1 ; \\
t^{\alpha-1}(1-s)^{\alpha-1}, \quad 0 \leq t \leq s \leq 1, \eta \leq s,
\end{array}\right.
$$

where $p(s)=1-\frac{\eta^{\alpha}}{\alpha}(1-s)$. Obviously, $G(t, s)$ is continuous on $[0,1] \times[0,1]$. 
Lemma 4.1 [35] Let $3<\alpha \leq 4$. Then the Green function $G(t, s)$ defined by (4.2) has the following properties:

(1) $G(t, s) \geq 0,(t, s) \in[0,1] \times[0,1]$

(2) $\frac{\eta^{\alpha}}{\Gamma(\alpha)\left(\alpha-\eta^{\alpha}\right)} s(1-s)^{\alpha-1} h(t) \leq G(t, s) \leq \frac{(\alpha-1)\left(\alpha-\eta^{\alpha}\right)+4 \eta^{\alpha-1}}{\Gamma(\alpha)\left(\alpha-\eta^{\alpha}\right)}(1-s)^{\alpha-1} h(t), t, s \in[0,1]$, where $h(t)=t^{\alpha-1}$.

Theorem 4.2 Assume that the following conditions $\left(\mathrm{H}_{1}\right)-\left(\mathrm{H}_{5}\right)$ hold:

$\left(\mathrm{H}_{1}\right) f, g, k:[0,1] \times[0,+\infty) \times[0,+\infty) \rightarrow[0,+\infty)$ are continuous, with $f(t, 0,1) \not \equiv 0$, $g(t, 0,1) \not \equiv 0, k(t, 0,1) \not \equiv 0, t \in[0,1]$

$\left(\mathrm{H}_{2}\right)$ for any fixed $t \in[0,1]$ and $v \in[0,+\infty), f(t, u, v), g(t, u, v), k(t, u, v)$ are increasing in $u \in[0,+\infty)$; for any fixed $t \in[0,1]$ and $u \in[0,+\infty), f(t, u, v), g(t, u, v), k(t, u, v)$ are decreasing in $v \in[0,+\infty)$;

$\left(\mathrm{H}_{3}\right)$ for $\lambda \in(0,1), t \in[0,1], u, v \in[0,+\infty), g\left(t, \lambda u, \lambda^{-1} v\right) \geq \lambda g(t, u, v)$; for any $\lambda \in(0,1), t \in$ $[0,1], u, v \in[0,+\infty)$, there exists $\varphi(\lambda) \in(\lambda, 1]$ such that $f\left(t, \lambda u, \lambda^{-1} v\right) \geq \varphi(\lambda) f(t, u, v)$; and for fixed $t \in[0,1], v \in[0,+\infty), k(t, \cdot, v)$ is concave; for fixed $t \in[0,1], u \in[0,+\infty)$, $k(t, u, \cdot)$ is convex;

$\left(\mathrm{H}_{4}\right)$ there exists $\frac{1}{2} \leq c \leq 1$ such that $k(s, \theta, \operatorname{lh}(t)) \geq c k(s, \operatorname{lh}(t), \theta), l \geq 1$;

$\left(\mathrm{H}_{5}\right)$ there exists a constant $\delta_{0}>0$, such that $g(t, u, v)+k(t, u, v) \leq \delta_{0} f(t, u, v), \forall t \in[0,1]$, $u, v \in[0,+\infty)$.

Then the problem (4.1) has a unique positive solution $u^{*}$ in $P$, which satisfies $\mu t^{\alpha-1} \leq$ $u^{*}(t) \leq \lambda t^{\alpha-1}$, where $\lambda>0, \mu>0$ are two real numbers, $t \in[0,1]$. Furthermore for any $x_{0}, y_{0} \in P_{h}$, by constructing successively the sequences

$$
\begin{aligned}
x_{n+1}(t)= & \int_{0}^{1} G(t, s) f\left(s, x_{n}(s), y_{n}(s)\right) d s+\int_{0}^{1} G(t, s) g\left(s, x_{n}(s), y_{n}(s)\right) d s \\
& +\int_{0}^{1} G(t, s) k\left(s, x_{n}(s), y_{n}(s)\right) d s, \quad n=0,1,2, \ldots, \\
y_{n+1}(t)= & \int_{0}^{1} G(t, s) f\left(s, y_{n}(s), x_{n}(s)\right) d s+\int_{0}^{1} G(t, s) g\left(s, y_{n}(s), x_{n}(s)\right) d s \\
& +\int_{0}^{1} G(t, s) k\left(s, y_{n}(s), x_{n}(s)\right) d s, \quad n=0,1,2, \ldots,
\end{aligned}
$$

we have $x_{n}(t) \rightrightarrows u^{*}, t \in[0,1]$ and $y_{n}(t) \rightrightarrows u^{*}(t), t \in[0,1]$.

Proof From [29], the problem (4.1) has an integral formulation given by

$$
u(t)=\int G(t, s)[f(s, u(s), u(s))+g(s, u(s), u(s))+k(s, u(s), u(s))] d s,
$$

where $\mathrm{G}(\mathrm{t}, \mathrm{s})$ is given as in $(4.2)$.

Define three operators $A, B, C: P \times P \rightarrow E$ by

$$
\begin{aligned}
& A(u, v)(t)=\int_{0}^{1} G(t, s) f(s, u(s), v(s)) d s \\
& B(u, v)(t)=\int_{0}^{1} G(t, s) g(s, u(s), v(s)) d s
\end{aligned}
$$




$$
C(u, v)(t)=\int_{0}^{1} G(t, s) k(s, u(s), v(s)) d s
$$

It is easy to prove that $u$ is the solution of the problem (4.1) if and only if $u=A(u, u)+$ $B(u, u)+C(u, u)$. From $\left(\mathrm{H}_{1}\right)$, we know that $A, B, C: P \times P \rightarrow P$. Further, it follows from $\left(\mathrm{H}_{2}\right)$ that $A, B, C$ are mixed monotone. For any $\lambda \in(0,1)$ and $u, v \in P$, by $\left(\mathrm{H}_{3}\right)$ we obtain

$$
\begin{aligned}
A\left(\lambda u, \lambda^{-1} v\right) & =\int_{0}^{1} G(t, s) f\left(s, \lambda u(s), \lambda^{-1} v(s)\right) d s \\
& \geq \varphi(\lambda) \int_{0}^{1} G(t, s) f(s, u(s), v(s)) d s \\
& =\varphi(\lambda) A(u, v)(t) .
\end{aligned}
$$

That is, $A\left(\lambda u, \lambda^{-1} v\right) \geq \varphi(\lambda) A(u, v)$ for $\lambda \in(0,1), u, v \in P$. So the operator $A$ satisfies (3.1). Also, for any $\lambda \in(0,1)$ and $u, v \in P$, from $\left(\mathrm{H}_{3}\right)$ we know that

$$
\begin{aligned}
B\left(\lambda u, \lambda^{-1} v\right) & =\int_{0}^{1} G(t, s) g\left(s, \lambda u(s), \lambda^{-1} v(s)\right) d s \\
& \geq \lambda \int_{0}^{1} G(t, s) g(s, u(s), v(s)) d s \\
& =\lambda B(u, v)(t) .
\end{aligned}
$$

That is, $B\left(\lambda u, \lambda^{-1} v\right) \geq \lambda B(u, v)$ for $\lambda \in(0,1), u, v \in P$. So the operator $A$ satisfies (3.2).

Now we prove that for fixed $v \in[0,+\infty), C(t, \cdot, v): P \rightarrow P$ is concave; for fixed $u \in$ $[0,+\infty), C(t, u, \cdot): P \rightarrow P$ is convex. For fixed $t \in(0,1), v \in[0,+\infty)$, for any $a \in(0,1)$, $u_{1}, u_{2} \in P$,

$$
\begin{aligned}
C\left(a u_{1}+(1-a) u_{2}, v\right)= & \int_{0}^{1} G(t, s) k\left(s, a u_{1}(s)+(1-a) u_{2}(s), v(s)\right) d s \\
\geq & \int_{0}^{1} G(t, s)\left(a k\left(s, u_{1}(s), v(s)\right)+(1-a) k\left(s, u_{2}(s), v(s)\right)\right) d s \\
= & a \int_{0}^{1} G(t, s) k\left(s, u_{1}(s), v(s)\right) d s \\
& +(1-a) \int_{0}^{1} G(t, s) k\left(s, u_{2}(s), v(s)\right) d s \\
= & a C\left(u_{1}(s), v(s)\right)+(1-a) C\left(u_{2}(s), v(s)\right),
\end{aligned}
$$

so for fixed $v \in[0,+\infty), C(t, \cdot, v): P \rightarrow P$ is concave; for fixed $t \in(0,1), u \in[0,+\infty)$, for any $a \in(0,1), v_{1}, v_{2} \in P$,

$$
\begin{aligned}
C\left(u, a v_{1}+(1-a) v_{2}\right) & =\int_{0}^{1} G(t, s) k\left(s, u(s), a v_{1}(s)+(1-a) v_{2}(s)\right) d s \\
& \leq \int_{0}^{1} G(t, s)\left(a k\left(s, u(s), v_{1}(s)\right)+(1-a) k\left(s, u(s), v_{2}(s)\right)\right) d s \\
& =a \int_{0}^{1} G(t, s) k\left(s, u(s), v_{1}(s)\right) d s
\end{aligned}
$$




$$
\begin{aligned}
& +(1-a) \int_{0}^{1} G(t, s) k\left(s, u(s), v_{2}(s)\right) d s \\
= & a C\left(u(s), v_{1}(s)\right)+(1-a) C\left(u(s), v_{2}(s)\right),
\end{aligned}
$$

so for fixed $u \in[0,+\infty), C(t, u, \cdot): P \rightarrow P$ is convex.

Then we show that $A(h, h) \in P_{h}, B(h, h) \in P_{h}$, and $C(h, h) \in P_{h}$. In fact, from (4.2) and Lemma 4.1

$$
\begin{aligned}
A(h, h)(t) & =\int_{0}^{1} G(t, s) k(s, f(s), f(s)) d s \\
& \leq \frac{(\alpha-1)\left(\alpha-\eta^{\alpha}\right)+4 \eta^{\alpha-1}}{\Gamma(\alpha)\left(\alpha-\eta^{\alpha}\right)} h(t) \int_{0}^{1}(1-s)^{\alpha-1} f(s, 1,0) d s \\
A(h, h)(t) & =\int_{0}^{1} G(t, s) f(s, h(s), h(s)) d s \geq \frac{\eta^{\alpha}}{\Gamma(\alpha)\left(\alpha-\eta^{\alpha}\right)} h(t) \int_{0}^{1} s(1-s)^{\alpha-1} f(s, 0,1) d s .
\end{aligned}
$$

From $\left(\mathrm{H}_{2}\right)$, we have

$$
f(s, 1,0) \geq f(s, 0,1) \not \equiv 0, \quad \forall s \in[0,1]
$$

so

$$
\int_{0}^{1} f(s, 1,0) d s \geq \int_{0}^{1} f(s, 0,1) d s>0
$$

and consequently $A(h, h) \in P_{h}$. Similarly,

$$
\begin{aligned}
& \frac{\eta^{\alpha}}{\Gamma(\alpha)\left(\alpha-\eta^{\alpha}\right)} h(t) \int_{0}^{1} s(1-s)^{\alpha-1} g(s, 0,1) d s \\
& \quad \leq B(h(t), h(t)) \leq \frac{(\alpha-1)\left(\alpha-\eta^{\alpha}\right)+4 \eta^{\alpha-1}}{\Gamma(\alpha)\left(\alpha-\eta^{\alpha}\right)} h(t) \int_{0}^{1} g(s, 1,0)(1-s)^{\alpha-1} d s,
\end{aligned}
$$

from $g(t, 0,1) \not \equiv 0$, we have $B(h, h) \in P_{h}$.

$$
\begin{aligned}
& \frac{\eta^{\alpha}}{\Gamma(\alpha)\left(\alpha-\eta^{\alpha}\right)} h(t) \int_{0}^{1} s(1-s)^{\alpha-1} k(s, 0,1) d s \\
& \quad \leq C(h(t), h(t)) \leq \frac{(\alpha-1)\left(\alpha-\eta^{\alpha}\right)+4 \eta^{\alpha-1}}{\Gamma(\alpha)\left(\alpha-\eta^{\alpha}\right)} h(t) \int_{0}^{1}(1-s)^{\alpha-1} k(s, 1,0) d s,
\end{aligned}
$$

from $k(t, 0,1) \not \equiv 0$, we have $C(h, h) \in P_{h}$. Hence the condition (4) of Theorem 3.1 is satisfied. In the following we show that the condition (6) of Theorem 3.1 is satisfied. From $\left(\mathrm{H}_{4}\right)$, there exists $\frac{1}{2} \leq c \leq 1$ such that

$$
\begin{aligned}
C(\theta, \operatorname{lh}(t)) & =\int_{0}^{1} G(t, s) k(s, \theta, \operatorname{lh}(s)) d s \geq c \int_{0}^{1} G(t, s) k(s, \ln (s), \theta) d s \\
& =c C(\ln (t), \theta), \quad l \geq 1 .
\end{aligned}
$$


For $u, v \in P$, from $\left(\mathrm{H}_{5}\right)$,

$$
\begin{aligned}
B(u, v)(t)+C(u, v)(t) & =\int_{0}^{1} G(t, s)[g(s, u(s), v(s))+k(s, u(s), v(s))] d s \\
& \leq \delta_{0} \int_{0}^{1} G(t, s) f(s, u(s), v(s)) d s=\delta_{0} A(u, v)(t) .
\end{aligned}
$$

Then we get $B(u, v)+C(u, v) \leq \delta_{0} A(u, v), u, v \in P$. So the conclusions of Theorem 4.2 follow from Theorem 3.1.

Example 4.1 Consider the boundary value problem

$$
\left\{\begin{array}{l}
D_{0+}^{\frac{7}{2}} u(t)=t^{2}+(u+1)^{\frac{1}{2}}+(u+1)^{\frac{1}{3}}+(v+1)^{-1}+(v+1)^{-\frac{1}{5}}-\frac{1}{4} e^{-u}+\frac{1}{4} e^{-v}+3 \\
\quad 0<t<1 \\
u(0)=u^{\prime}(0)=u^{\prime \prime}(0)=0 \\
u(1)=\int_{0}^{\frac{1}{2}} u(s) d s .
\end{array}\right.
$$

Consider the functions $f, g, k:[0,1] \times[0,+\infty) \times[0,+\infty) \rightarrow[0,+\infty)$, defined by

$$
\begin{aligned}
& f(t, x, y)=t^{2}+1+(x+1)^{\frac{1}{2}}+(y+1)^{-\frac{1}{5}}, \quad t \in[0,1], x, y \geq 0, \\
& g(t, x, y)=t^{2}+(x+1)^{\frac{1}{3}}+(y+1)^{-1}, \quad t \in[0,1], x, y \geq 0, \\
& k(t, x, y)=-t^{2}+2-\frac{1}{4} e^{-x}+\frac{1}{4} e^{-y}, \quad t \in[0,1], x, y \geq 0 .
\end{aligned}
$$

Then (4.3) is equivalent to

$$
\left\{\begin{array}{l}
D_{0+}^{\frac{7}{2}} u(t)=f(t, u, u)+g(t, u, u)+k(t, u, u), \quad 0<t<1, \\
u(0)=u^{\prime}(0)=u^{\prime \prime}(0)=0 \\
u(1)=\int_{0}^{\frac{1}{2}} u(s) d s .
\end{array}\right.
$$

Let us check that all the required conditions of Theorem 4.2 are satisfied.

(1) Clearly, the functions $f, g, k:[0,1] \times[0,+\infty) \times[0,+\infty) \rightarrow[0,+\infty)$ are continuous with $f(t, 0,1) \not \equiv 0, g(t, 0,1) \not \equiv 0, k(t, 0,1) \not \equiv 0$.

(2) We observe easily that for fixed $t \in[0,1]$ and $y \in[0,+\infty), f(t, x, y), g(t, x, y), k(t, x, y)$ are increasing in $x \in[0,+\infty)$; for fixed $t \in[0,1]$ and $x \in[0,+\infty), f(t, x, y), g(t, x, y), k(t, x, y)$ are decreasing in $y \in[0,+\infty)$.

(3) For all $\lambda \in(0,1), t \in[0,1]$, and $x \geq 0, y \geq 0$, taking $\varphi(\lambda)=\lambda^{\frac{1}{2}} \in(\lambda, 1)$, we have

$$
\begin{aligned}
f\left(t, \lambda x, \lambda^{-1} y\right) & =t^{2}+1+(\lambda x+1)^{\frac{1}{2}}+\left(\lambda^{-1} y+1\right)^{-\frac{1}{5}} \\
& \geq t^{2}+1+(\lambda x+\lambda)^{\frac{1}{2}}+\left(\lambda^{-1} y+\lambda^{-1}\right)^{-\frac{1}{5}} \\
& =t^{2}+1+\lambda^{\frac{1}{2}}(x+1)^{\frac{1}{2}}+\lambda^{\frac{1}{5}}(y+1)^{-\frac{1}{5}} \\
& \geq \varphi(\lambda)\left[t^{2}+1+(x+1)^{\frac{1}{2}}+(y+1)^{-\frac{1}{5}}\right] \\
& =\varphi(\lambda) f(t, x, y) .
\end{aligned}
$$


For all $\lambda \in(0,1), t \in[0,1]$, and $x \geq 0, y \geq 0$, we have

$$
\begin{aligned}
g\left(t, \lambda x, \lambda^{-1} y\right) & =t^{2}+(\lambda x+1)^{\frac{1}{2}}+\left(\lambda^{-1} y+1\right)^{-1} \\
& \geq t^{2}+(\lambda x+\lambda)^{\frac{1}{2}}+\left(\lambda^{-1} y+\lambda^{-1}\right)^{-1} \\
& \geq \lambda t^{2}+\lambda^{\frac{1}{2}}(x+1)^{\frac{1}{2}}+\lambda(y+1)^{-1} \\
& \geq \lambda\left[t^{2}+(x+1)^{\frac{1}{2}}+(y+1)^{-1}\right] \\
& =\lambda g(t, x, y) .
\end{aligned}
$$

It is easy to prove that

$$
k_{u u}^{\prime \prime}(t, x, y)=-\frac{1}{4} e^{-x}<0, \quad k_{v v}^{\prime \prime}(t, x, y)=\frac{1}{4} e^{-y}>0 .
$$

So for fixed $t \in(0,1), y \in[0,+\infty), k(t, \cdot y)$ is concave; for fixed $t \in(0,1), x \in[0,+\infty)$, $k(t, x, \cdot)$ is convex.

(4) For all $s \in(0,1), y \in[0,+\infty)$, taking $c=\frac{8}{15}$, it is easy to prove

$$
k(s, \theta, y)=\left(1-s^{2}\right)+\left(\frac{3}{4}+\frac{1}{4} e^{-y}\right) \geq c\left[\left(1-s^{2}\right)+\left(\frac{5}{4}-\frac{1}{4} e^{-y}\right)\right]=c k(s, y, \theta) .
$$

(5) Taking $\delta_{0}=3$, then

$$
\begin{aligned}
g(t, x, y)+k(t, x, y) & =\left[t^{2}+(x+1)^{\frac{1}{3}}+(y+1)^{-1}\right]+\left[-t^{2}+2-\frac{1}{4} e^{-x}+\frac{1}{4} e^{-y}\right] \\
& =(x+1)^{\frac{1}{3}}+(y+1)^{-\frac{1}{4}}+2-\frac{1}{4} e^{-x}+\frac{1}{4} e^{-y} \\
& \leq 3\left[t^{2}+1+(x+1)^{\frac{1}{2}}+(y+1)^{-\frac{1}{5}}\right] \\
& =3 f(t, x, y) \\
& =\delta_{0} f(t, x, y) .
\end{aligned}
$$

Thus we proved that all the hypotheses of Theorem 4.2 are satisfied. Then we deduce that (4.3) has one and only one positive solution $x^{*} \in P_{h}$, where $h(t)=t^{\frac{5}{2}}, t \in[0,1]$.

\section{Competing interests}

The authors declare that there is no conflict of interests regarding the publication of this paper.

\section{Authors' contributions}

All authors contributed equally and significantly in writing this article. All authors read and approved the final manuscript.

\section{Author details}

'School of Mathematical Sciences, Qufu Normal University, Qufu, Shandong 273165, People's Republic of China. ${ }^{2}$ Department of Mathematics and Statistics, Curtin University, Perth, WA 6845, Australia. ${ }^{3}$ School of Statistics and Mathematics, Zhongnan University of Economics and Law, Wuhan, 430073, People's Republic of China.

\section{Acknowledgements}

The authors were supported financially by the National Natural Science Foundation of China $(11371221,11571296)$, the Specialized Research Foundation for the Doctoral Program of Higher Education of China (20123705110001), and the Program for Scientific Research Innovation Team in Colleges and Universities of Shandong Province. 


\section{References}

1. Guo, D, Lakskmikantham, V: Coupled fixed points of nonlinear operators with applications. Nonlinear Anal. 11(5), 623-632 (1987)

2. Lakskmikantham, V, Ćirić, L: Coupled fixed point theorems for nonlinear contractions in partially ordered metric spaces. Nonlinear Anal. 70, 4341-4349 (2009)

3. Li, K, Liang, J, Xiao, T: New existence and uniqueness theorems of positive fixed points for mixed monotone operators with perturbation. J. Math. Anal. Appl. 328, 753-766 (2007)

4. Nussbaum, R: Iterated nonlinear maps and Hilbert's projective metric. Mem. Am. Math. Soc. 75, 391 (1988)

5. Podulubny, I: Fractional Differential Equations. Mathematics in Science and Engineering. Academic Press, New York (1999)

6. Sun, Y: A fixed point theorem for mixed monotone operator with applications. J. Math. Anal. Appl. 156, 240-252 (1991)

7. $\mathrm{Wu}, \mathrm{Y}, \mathrm{Li}, \mathrm{G}$ : On the fixed point existence and uniqueness theorems of mixed monotone operators and applications. Acta Math. Sin. 46(1), 161-166 (2003) (in Chinese)

8. Wu, Y, Liang, Z: Existence and uniqueness of fixed points for mixed monotone operators with applications. Nonlinear Anal. 65, 1913-1924 (2006)

9. Zhang, S, Ma, Y: Coupled fixed points for mixed monotone condensing operators and an existence theorem of the solution for a class of functional equations arising in dynamic programming. J. Math. Anal. Appl. 160, 468-497 (1991)

10. Zhang, Z, Wang, K: On fixed point theorems of mixed monotone operators and applications. Nonlinear Anal. 70 , 3279-3284 (2009)

11. Chen, $Y$ : The existence of a fixed point for the sum of two monotone operators. Positivity $12,643-652$ (2008)

12. Zhang, Z: Fixed point theorems of mixed monotone operators and its applications. Acta Math. Sin. 41, 1121-1126 (1998)

13. Zhai, $\mathrm{C}, \mathrm{Hao}, \mathrm{M}$ : Fixed point theorems for mixed monotone operators with perturbation and applications to fractional differential equation boundary value problems. Nonlinear Anal. 75, 2542-2551 (2012)

14. Zhai, C, Anderson, DR: A sum operator equation and applications to nonlinear elastic beam equations and Lane-Emden-Fowler equations. J. Math. Anal. Appl. 375, 388-400 (2011)

15. Chen, Y: Thompson's metric and mixed monotone operators. J. Math. Anal. Appl. 177, 31-37 (1993)

16. Guo, D: Fixed points of mixed monotone operators with application. Appl. Anal. 34, 215-224 (1988)

17. Hilger, S: Analysis on measure chains - a unified approach to continuous and discrete calculus. Results Math. 18 18-56 (1990)

18. Lian, $X, L i, Y$ : Fixed point theorems for a class of mixed monotone operators with applications. Nonlinear Anal. 67, 2752-2762 (2007)

19. Liang, Z, Zhang, L, Li, S: Fixed point theorems for a class of mixed monotone operators. Z. Anal. Anwend. 22(3), 529-542 (2003)

20. Wang, W, Liu, X, Cheng, S: Fixed points for mixed monotone operators and applications. Nonlinear Stud. 14(2), 189-204 (2007)

21. Zhai, C, Zhang, L: New fixed point theorems for mixed monotone operators and local existence-uniqueness of positive solutions for nonlinear boundary value problems. J. Math. Anal. Appl. 382, 594-614 (2011)

22. Zhang, Z: New fixed point theorems of mixed monotone operators and applications. J. Math. Anal. Appl. 204 307-319 (1996)

23. Zhao, Z: Existence and uniqueness of fixed points for some mixed monotone operators. Nonlinear Anal. 73 1481-1490 (2010)

24. Deimling, K: Nonlinear Functional Analysis. Springer, Berlin (1985)

25. Guo, D, Lakskmikantham, V: Nonlinear Problems in Abstract Cones. Academic Press, New York (1988),

26. Lei, $P$, Lin, $X$, Jiang, D: Existence and uniqueness of positive solutions for singular nonlinear elliptic boundary value problems. Nonlinear Anal. 69, 2773-2779 (2008)

27. El-Sayed, AMA: Nonlinear functional differential equations of arbitrary orders. Nonlinear Anal. 33, 181-186 (1998)

28. Harjiani, J, López, B, Sadarangani, K: Fixed point theorems for mixed monotone operators and applications to integral equations. Nonlinear Anal. 74, 1749-1760 (2001)

29. Jiang, M, Zhong, S: Successively iterative method for fractional differential equations with integral boundary conditions. Appl. Math. Lett. 38, 94-99 (2014)

30. Kilbas, AA, Srivastava, HM, Trujillo, JJ: Theory and Applications of Fractional Differential Equations. North-Holland Mathematics Studies, vol. 204. Elsevier, Amsterdam (2006)

31. Lin, X, Jiang, D, Li, X: Existence and uniqueness of solutions for singular fourth-order boundary value problems. Comput. Appl. Math. 196, 155-161 (2006)

32. Miller, KS, Ross, B: An Introduction to the Fractional Calculus and Fractional Differential Equations. Wiley, New York (1993)

33. Samko, SG, Kilbas, AA, Marichev, OI: Fractional integral and derivatives. In: Theory and Applications. Gordon \& Breach, Yverdon (1993)

34. Yuan, C, Jiang, D, Zhang, Y: Existence and uniqueness of solutions for singular higher order continuous and discrete boundary value problems. Bound. Value Probl. 2008, 123823 (2008)

35. Xu, X, Jiang, D, Yuan, C: Multiple positive solutions for boundary value problem of nonlinear fractional differential equation. Nonlinear Anal. 71, 4676-4688 (2009) 